\title{
Vascular branching geometry relating to portal hypertension: a study of liver microvasculature in cirrhotic rats by X-ray phase- contrast computed tomography
}

\author{
Mengyu Sun ${ }^{1 \#}$, Wenjuan Lv ${ }^{1 \#}$, Xinyan Zhao ${ }^{2}$, Lili Qin ${ }^{1}$, Yuqing Zhao ${ }^{1}$, Xiaohong Xin ${ }^{1}$, Jianbo Jian ${ }^{3}$, \\ Xiaodong Chen ${ }^{4}$, Chunhong $\mathrm{Hu}^{1}$ \\ ${ }^{1}$ School of Biomedical Engineering and Technology, Tianjin Medical University, Tianjin 300070, China; ${ }^{2}$ Liver Research Center, Beijing \\ Friendship Hospital, Capital Medical University, Beijing 100050, China; ${ }^{3}$ Radiation Oncology Department, Tianjin Medical University General \\ Hospital, Tianjin 300070, China; ${ }^{4}$ Key Laboratory of Optoelectronic Information Technology, Ministry of Education (Tianjin University), \\ Tianjin 300072, China
}

\#These authors contributed equally to this work.

Correspondence to: Chunhong Hu. School of Biomedical Engineering and Technology, Tianjin Medical University, No. 22 Qixiangtai Road, Tianjin 300070, China. Email: chunhong_hu@hotmail.com.

\begin{abstract}
Background: Portal hypertension is one of the major complications of cirrhosis. The changes in hepatic microvasculature are considered as critical pathophysiological characteristics of portal hypertension. X-ray phase-contrast computed tomography (PCCT) is a new imaging technique that can detect liver vessels at a micrometric level without contrast agents.

Methods: In this study, male Sprague-Dawley rats with liver cirrhosis induced by carbon tetrachloride $\left(\mathrm{CCl}_{4}\right)$ or bile duct ligation (BDL) were investigated with PCCT. The portal pressures of rats were recorded before euthanasia. The branch angle and Murray's deviation (MD) were measured based on the branching geometry of the three-dimensional (3D) microvasculature of liver cirrhosis in rats. Statistical analyses were performed to determine the correlation between branching geometry and portal pressure in liver fibrosis.

Results: The results demonstrated that the branch angle and $\mathrm{MD}$ significantly increased in the $\mathrm{CCl}_{4}$ model and BDL model compared with their corresponding normal group or sham group. The portal pressure was significantly correlated with the branching morphologic features (all $\mathrm{R} \geq 0.761$ and $\mathrm{P}<0.01$ ).

Conclusions: The branch angle and MD could accurately distinguish portal pressure in cirrhotic rats, suggesting that branching geometric characteristics of the microvasculature may be a promising marker in the prognosis of portal hypertension in liver cirrhosis.
\end{abstract}

Keywords: Portal hypertension; Murray's deviation (MD); branch angle; three-dimensional visualization (3D visualization); X-ray phase-contrast computed tomography (X-ray PCCT)

Submitted May 20, 2019. Accepted for publication Oct 30, 2019.

doi: 10.21037/qims.2019.11.04

View this article at: http://dx.doi.org/10.21037/qims.2019.11.04

\section{Introduction}

Portal hypertension, defined as portal pressure $\geq 10 \mathrm{mmHg}$, is regarded as one of the most major complications of cirrhosis and contributes to the occurrences of ascites, hypersplenism, and esophageal varices, resulting in the death of cirrhosis patients (1). Portal pressure is highly correlated with the progression of cirrhosis and patient prognosis. Until now, hepatic venous pressure gradient (HVPG), which measures the difference between wedge hepatic venous pressure and free hepatic venous pressure, 
has remained the standard method for the measurement of portal hypertension (2). Nevertheless, this invasive method relies on the experiences of observers, which hinders its widespread application. Thus, there is a strong demand for surrogate markers of HVPG that can detect portal hypertension efficiently and intelligently. Earlier studies have found a significant relationship between fibrosis area and portal hypertension, which is consistent with clinical diagnosis parameters. Recently, the changes in hepatic microvasculature are considered as an essential pathophysiological characteristic of portal hypertension. Microvascular changes in liver fibrosis can be utilized to investigate the formation mechanisms in resistance to blood flow and elevate portal pressure directly. Some researchers have reported obvious microvascular and hemodynamic changes in liver cirrhosis or portal hypertension (3-5).

Additionally, the branching geometry of the vasculature is investigated in normal liver based on Murray's law, and the study demonstrates that the hepatic artery and portal vein strongly adheres to Murray's cube law. In other words, the vascular system may diverge from the law in a diseased liver, which may result in Murray's deviation (MD) and an abnormal branch angle (6). In this study, we hypothesized that vascular morphologic features might be used to distinguish portal hypertension. Therefore, a threedimensional (3D) structure of liver vessels needs to be obtained to quantize microvasculature morphology features for evaluating portal pressure.

Imaging techniques can provide detailed observations of vessels. However, clinical imaging modalities, such as ultrasound, computed tomography (CT), and magnetic resonance imaging (MRI), are unable to present microvascular changes due to their poor spatial resolution and insufficient contrast. Fluorescence labeling of vascular endothelium is the conventional method for studying liver microvasculature, and it can provide detailed observations of vessel branches. However, this technique only acquires a given cross-section of the vascular architecture, which often yields discontinuous and incomplete information. Moreover, fluorescence labeling of vascular endothelium destroys tissue integrity and has a complex sample preparation process and limited vision for analysis. Some techniques, such as optical coherence tomography (OCT), scanning electron microscopy (SEM) or transmission electron microscopy (TEM), can provide high-resolution images at the micron or even the nanometer scale, but they are limited to the surface or shallow depths $(7,8)$. Limited by the weak absorption between the soft tissues, the vascular structure is not easily detected via absorption-based CT. The absorption-based CT with contrast agent is regarded as an effective method to study the bifurcation of blood vessels (9). However, due to the insufficient spatial imaging resolution, the CT angiography technique is incapable of detecting of small vascular malformations with diameters of less than $200 \mu \mathrm{m}$. In advanced stages of cirrhosis, the vessels may become leaky, and the contrast agents can penetrate through the damaged parts of the vessel walls, which may cause a misleading result. Considering the abovementioned limitation, a high-resolution imaging technique for $3 \mathrm{D}$ visualization of the microvascular structure of the liver in rats is highly desirable. Compared to the absorption-based $\mathrm{CT}$ angiography technique, $\mathrm{X}$-ray phase-contrast computed tomography imaging (PCCT) is a phase-based imaging technique, which is sensitive to the refraction (i.e., "phase shift") of X-rays in matter. Since phase variations can be two to three orders of magnitude higher than the absorption ones $(10,11)$, PCCT enables the superior depiction of microstructural details, and can ascertain the quantitative parameters of the microvascular morphology without the use of contrast agents. Hence, this technique has been applied in the analyses and assessment of the changes of the organ tissues in different diseases, such as portal venous diseases, coronary atherosclerosis, spinal cord injury, and pulmonary fibrosis (12-15). Indeed, several studies have achieved 3D visualization of the microvasculature of liver fibrosis, and qualitative descriptions and quantitative results revealed the $3 \mathrm{D}$ diagnostic value of $\operatorname{PCCT}(3,16,17)$.

Cirrhosis induced by carbon tetrachloride $\left(\mathrm{CCl}_{4}\right)$ and bile duct ligation (BDL) in rats is widely utilized, and they represent experimental models of human chronic toxic cirrhosis and biliary cirrhosis, respectively. In this study, the liver cirrhosis models induced via $\mathrm{CCl}_{4}$ and $\mathrm{BDL}$ were used, and the corresponding portal pressures were measured and recorded for further analysis. The $3 \mathrm{D}$ liver microvasculature was obtained by PCCT, and the branch angle and MD were measured. The purpose of this work was to study the potential of vascular branching geometric features in evaluating portal hypertension in cirrhosis.

\section{Methods}

\section{Sample preparation}

All animal experiments were carried out with the approval of the Research Ethics Committee of Beijing Friendship Hospital, Capital Medical University, China. Twenty-four 
male Sprague-Dawley (SD) rats (8 weeks old, weighing $180-220 \mathrm{~g}$ ) used for all experiments were maintained in a temperature-controlled room $\left(23 \pm 2{ }^{\circ} \mathrm{C}, 55 \% \pm 10 \%\right.$ humidity $)$ with a 12-hour light-dark cycle and free access to water and food. In this study, cirrhosis was induced via $\mathrm{CCl}_{4}$ or BDL. All rats were randomly divided into four groups as follows: the $\mathrm{CCl}_{4}$ control group (the control group), the $\mathrm{CCl}_{4}$ group, the sham operation group (the sham group) and the BDL group (each group with 6 rats). The rats of $\mathrm{CCl}_{4}$ group were administered with $2 \mathrm{~mL}$ of $\mathrm{CCl}_{4} /$ olive oil (4:6, v/v) per $\mathrm{kg}$ body weight by intraperitoneal (IP) injection and twice per week for up to 12 weeks to establish the cirrhosis model. In the BDL group, the common bile duct (CBD) was stripped and double-ligated with 4.0 silk ligatures after anesthesia with phenobarbital and midline laparotomy.

Meanwhile, in the sham group, the CBD was simply exposed without ligation. After 12 and 4 weeks of modeling, a high degree of micronodular cirrhosis and biliary cirrhosis were established, respectively. The measurements of portal pressure were performed as described below. A catheter (ID $0.86 \mathrm{~mm}$, OD $1.27 \mathrm{~mm}$ ) was implanted into the portal vein in a rat under anesthesia condition (1). A sensitive pressure transducer (BL-420S Physiological Systems, Taimeng Instruments, Chengdu, China) was connected with this catheter to continuously record portal pressure for a 10-minute stabilization period $(4,18)$. Then all rats from the $\mathrm{CCl}_{4}$ model and BDL model were euthanized with a dose of $800 \mathrm{mg} / \mathrm{kg}$ of sodium pentobarbital at 12 and 4 weeks, respectively (19). After removal, all livers were rinsed by phosphate buffer and perfused with saline $0.9 \%$ to clear the blood vessels, then fixed in $10 \%$ neutral buffered formalin solution for follow-up experiments.

After imaging, the samples were embedded in paraffin, cut into 4- $\mu \mathrm{m}$-thick slides, and stained with Sirius Red to visualize collagenous tissues. The histological images were then analyzed using Image-Pro Plus software (version 6.0; Media Cybernetics, Bethesda, MD, USA). Areas of fibrosis were assessed in 20 randomly selected non-overlapping areas for each group at $40 \times$ magnification. The fibrosis area was expressed as the relative percentage between the area of fibrous tissue and the total area of liver tissue.

\section{Image acquisition}

High-solution images were obtained using in-line $\mathrm{X}$-ray phase-contrast imaging (IL-PCI) at the beamline BL13W1 of the Shanghai Synchrotron Radiation Facility (SSRF) in China, which is focused on biological imaging and biomedical application. The imaging schematic diagram of PCCT is represented in Figure 1. In this experiment, the sample was fixed on the rotation stage, the $\mathrm{X}$-ray beam energy was set at $24 \mathrm{keV}$, and the sample-to-detector distance (SDD) was $80 \mathrm{~cm}$. The raw projections were acquired from each sample over $180^{\circ}$ in rotation steps of $0.35^{\circ}$ with a $12 \mathrm{~ms}$ exposure time, and an effective pixel size of $9 \times 9 \mu^{2}$ was achieved. The detailed experiment parameters are provided in Table S1. Additionally, 10 flatfield images and 10 dark-field images were obtained to avoid artifacts and noises.

When an X-ray passes through a sample, the obtained projection images display a mix of absorption information and phase information, and the $3 \mathrm{D}$ complex refractive index distribution can be described as follows:

$$
n(x, y, z)=1-\delta(x, y, z)+i \beta(x, y, z)
$$

where the real part $\delta$ is the refractive index decrement that represents phase information; the imaginary $\beta$ is the absorption index; and $(\mathrm{x}, \mathrm{y}, \mathrm{z})$ are the spatial coordinates. For weakly absorbing objects (i.e., biological soft tissues), phase information plays a more significant role than the absorption information (20). Phase retrieval was used for obtaining phase information from the initial projections. In this study, the phase-attenuation duality Bronnikov algorithm (PAD-BA) was utilized to extract quantitative phase information (21). Then, the phase projection images, which only contained phase information, were utilized to reconstruct the $\mathrm{CT}$ slices based on a filtered back-projection (FBP) algorithm using PITRE software (applied by the BL13W1 station, SSRF). Finally, the 3D visualization and quantitative analysis of the microvasculature was performed using Amira software (Amira 6.3; FEI, Thermo Fisher Scientific, Bordeaux, France).

\section{Image analysis}

Murray's law indicates the optimal vascular bifurcation such that the cube of the diameter of a mother vessel equals the sum of the cubes of the diameters of the daughters (22), and this has been widely used to analyze the branching geometry of vessels $(23,24)$. In general, normal vessels obey Murray's law, but there may be an increase of MD due to the loss of an optimal circulatory system with the development of liver disease (6). MD can be described by the following formula:

$$
M D=\frac{\left|d_{0}^{3}-d_{1}^{3}-d_{2}^{3}\right|}{d_{0}^{3}}
$$




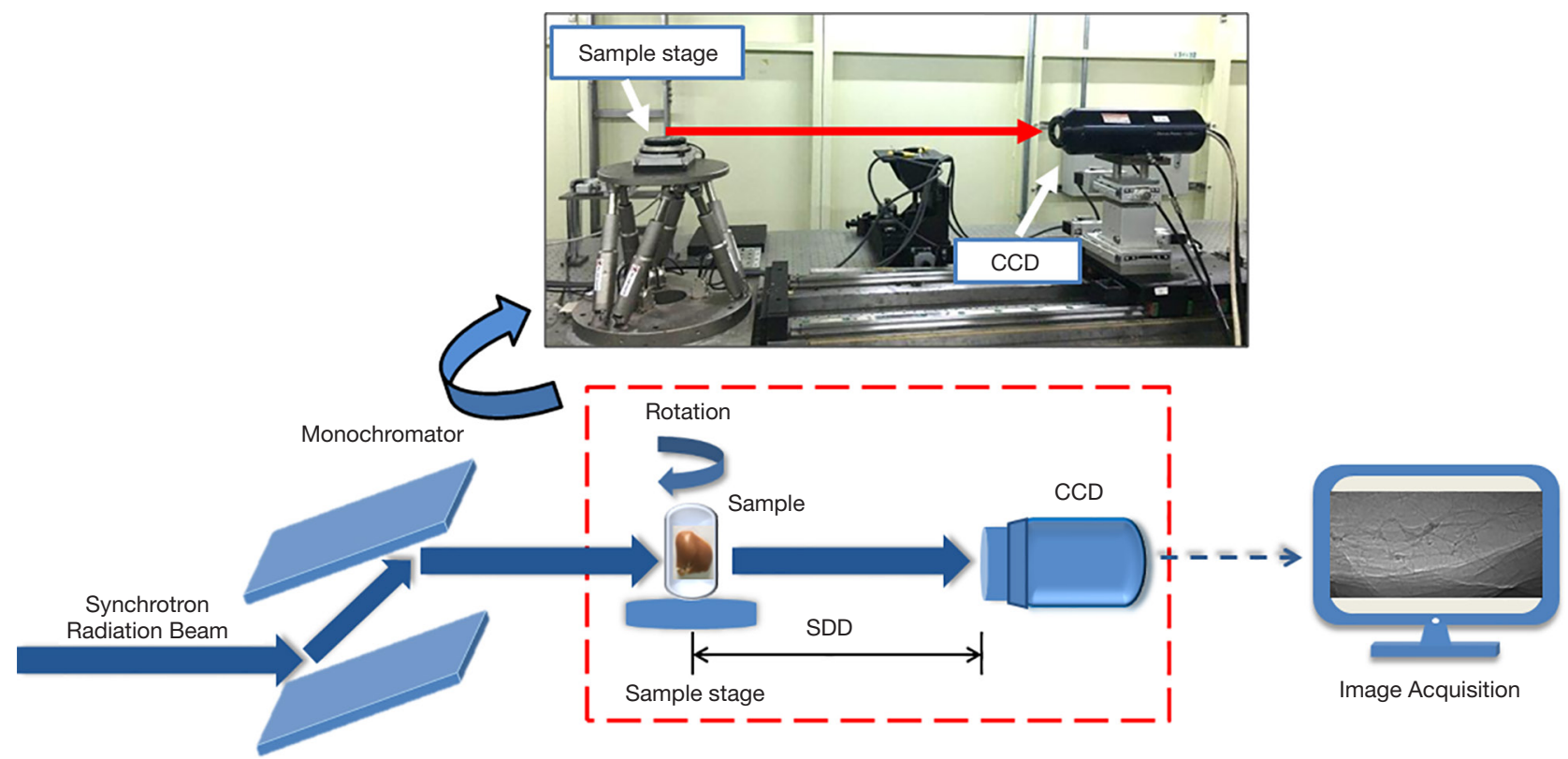

Figure 1 Schematic diagram of the PCI imaging station at BL13W1 in SSRF, China. The X-ray synchrotron radiation beam is projected on a sample that is fixed to the rotation sample stage. The inner information obtained from the transmitted beam is recorded by a detector located at a fixed distance to the sample stage. In the imaging progress, the sample rotates by 180 degrees to obtain different views of projections. SDD is the distance between sample and detector. PCI, phase-contrast imaging; SSRF, Shanghai Synchrotron Radiation Facility; SDD, sample-to-detector distance.

where $d_{0}$ represents the radius of the mother vessel, and $d_{2}$ and $d_{2}$ are the radii of daughters. In this study, MD was used to describe branching geometric features of the microvasculature in liver cirrhosis.

Also, the branch angle was an essential feature of vessel bifurcations, and the branch angle varied from approximately $60^{\circ}-75^{\circ}$ in the optimal circle system. The abnormal changes of the branch angle may indicate the deviation from the optimum circulation of blood. In our study, the branch angle $\theta$ was measured based on the extracted 3D vessel centerline, and it represented the mean plane angle between the vector segments adjacent to the bifurcation points.

Limited by the small field-of-view of the current PCI system, it is difficult to image the microvascular structure of the whole liver in rats, and here the traditional vascular classification methods are unavailable. As mentioned in previous studies, the traditional vascular classification methods are graded from main vessels to the vascular endings; that is, the main vessels are ranked as the first generation $(25,26)$. However, the vessel segments, which were used to evaluate MD values and branch angles in this study, started from the vascular endings rather than the main vessels, which made the traditional methods not applicable, and thus a new classification method was proposed. Contrary to the traditional vascular classification methods, this method ranked the branches from vascular endings to main vessels; namely, the vascular endings were ranked as the first generation (generation 1), and the ranking number of the branches increased with the increasing levels of branches. Based on the abovementioned method, 12 vascular segments were randomly selected in the control group, the $\mathrm{CCl}_{4}$ group, the sham group, and the BDL group respectively, and the 1st to 5 th generations of branches in the chosen vascular segments were utilized to calculate the branch angle and MD value. Finally, 60 branches in each group were selected to obtain the branch angle and MD values without consideration of vascular generations.

\section{Statistical analysis}

The data are presented as means \pm standard deviations $(\mathrm{SD})$. The statistical analyses were performed with SPSS software (version 19.0; IBM, Chicago, IL, USA). All data were analyzed by a two-tailed $t$-test to evaluate the differences 

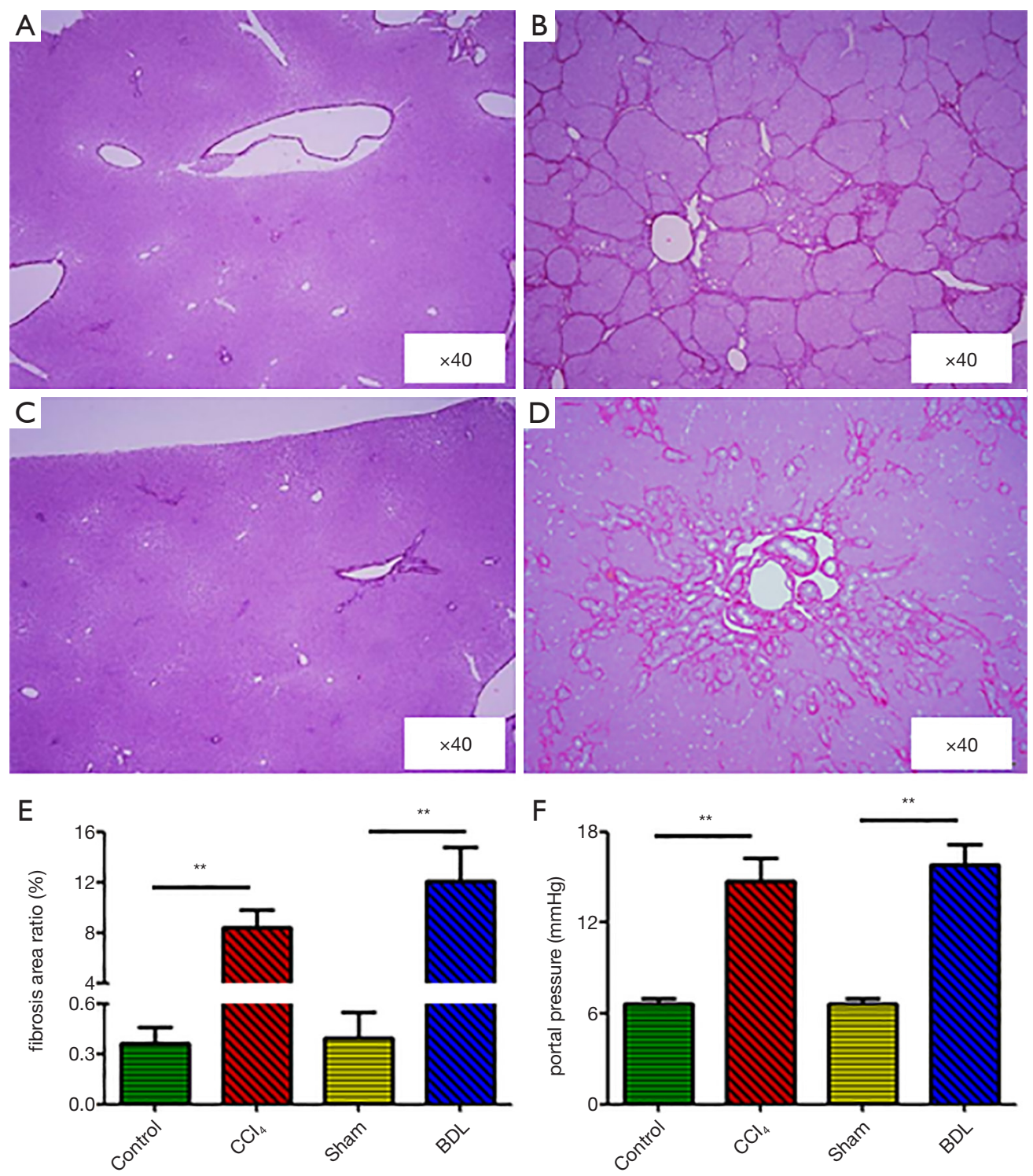

Figure 2 Histological sections and quantitative analyses of portal pressure and fibrosis area in two models. Histological sections stained by sirius red show representative images (A-D) of the control group, $\mathrm{CCl}_{4}$ group, sham group and BDL group, respectively. Portal pressure and fibrosis area $(\mathrm{E}, \mathrm{F})$ are summarized and shown for different groups. Values are represented by means $\pm \mathrm{SD}$. ${ }^{* *} \mathrm{P}<0.01$. $\mathrm{n}=6$ per group. The magnification of the histological section is $40 \times . \mathrm{CCl}_{4}$, carbon tetrachloride.

between the control groups and the corresponding cirrhosis groups. Correlations between the noted parameters were assessed using the Pearson correlation test. A P value of less than 0.05 was considered statistically significant, and the confidence interval (CI) was set at $95 \%$. Receiver operating characteristic (ROC) curve analyses were used to calculate the sensitivity and specificity of MD and branch angle in discriminating portal hypertension, defined as portal pressure $\geq 10 \mathrm{mmHg}(27)$.

\section{Results}

\section{Histopathological analysis}

The histological sections of rats from two models were stained by sirius red (Figure 2). In normal livers, collagen was detected in low amounts around portal tracts and 

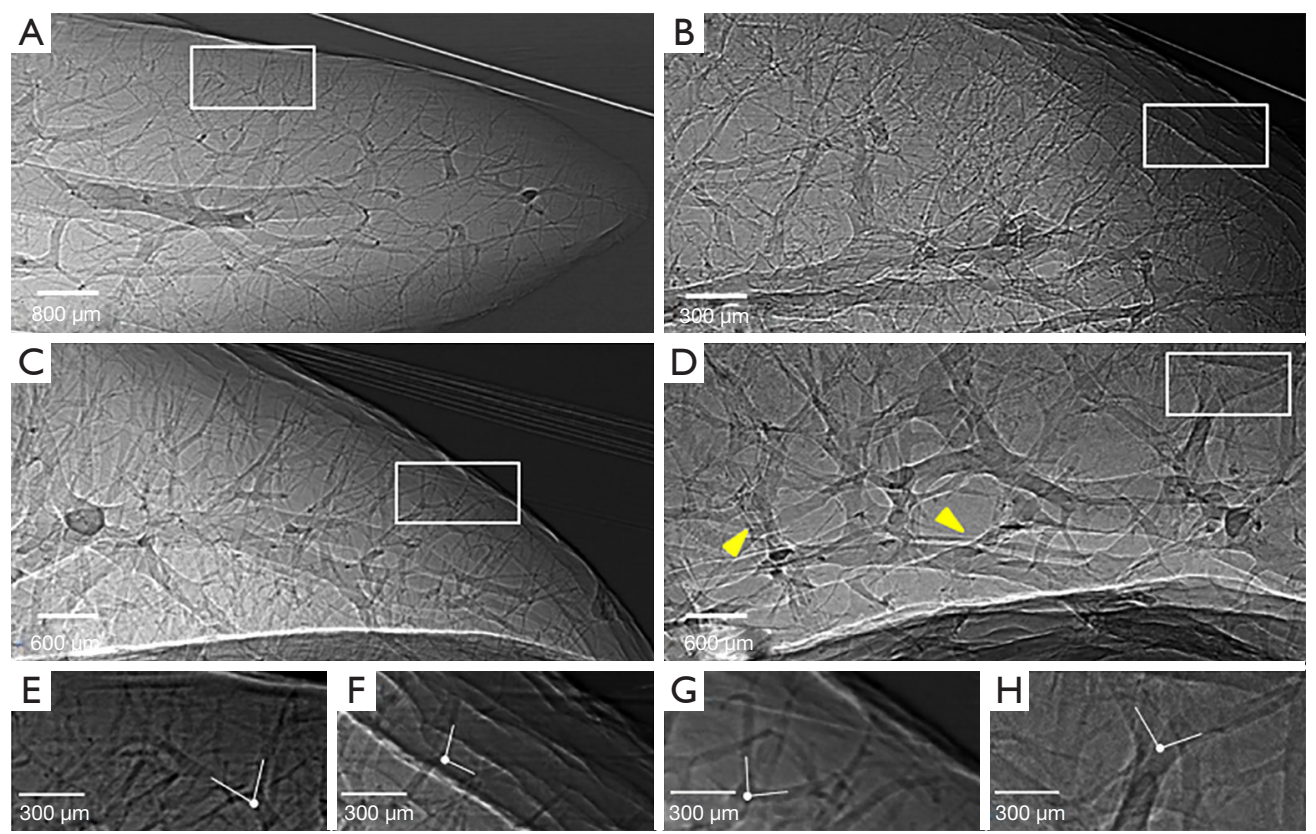

Figure 3 The PCI projection images in different groups, the representative images, which are obtained from the control group (A), the $\mathrm{CCl}_{4}$ group (B), the sham group (C) and the BDL group (D), characterize vascular changes in normal and cirrhotic liver samples. Panels (E), (F), (G) and $(\mathrm{H})$ are the amplified images surrounded by the white rectangle regions in (A), (B), (C) and (D), respectively. The bile duct is marked in (D) by yellow arrows. PCI, phase-contrast imaging; $\mathrm{CCl}_{4}$, carbon tetrachloride; BDL, bile duct ligation.

hepatic veins (Figure 2A,C). Compared to the normal group, the collagen fibrils in cirrhotic livers induced by $\mathrm{CCl}_{4}$ (Figure 2B) and BDL (Figure 2D) had apparent hyperplasia; the collagen septa destroyed the normal architecture of liver parenchyma, and pseudo-lobules were formed. Figure $2 E, F$ show the analyses of portal pressure and fibrosis area, respectively. In the $\mathrm{CCl}_{4}$ group, the fibrosis area $(8.36 \% \pm 1.49 \%)$ and portal pressure $(14.40 \pm 1.64 \mathrm{mmHg})$ are both markedly higher than in the control group $(0.36 \% \pm 0.1 \%$ and $\mathrm{P}<0.01,6.82 \pm 0.17 \mathrm{mmHg}$ and $\mathrm{P}<0.01$, respectively). In the BDL group, the fibrosis area and portal pressure are $12.02 \% \pm 2.73 \%$ and $16.01 \pm 1.50 \mathrm{mmHg}$, respectively, which significantly increased compared with the sham group $(0.39 \% \pm 0.15 \%$ and $\mathrm{P}<0.01,6.60 \pm 0.32 \mathrm{mmHg}$ and $\mathrm{P}<0.01$, respectively). The cirrhotic rats had a portal pressure $\geq 10 \mathrm{mmHg}$, which is defined as significant portal hypertension, and rats in the normal group or sham group had portal pressure $<10 \mathrm{mmHg}$.

\section{Projection images of liver cirrbosis samples}

The PCI projection images in different groups are shown in Figure 3. The vessels with a diameter of tens of micrometers were clearly distinguished from the surrounding soft tissues. The vessels (Figure $3 A, C$ ) in the normal livers appeared regular and ordered, while in the cirrhotic livers, the vessels (Figure $3 B, D$ ) became jumbled. As shown in the enlarged images of white rectangle regions (Figure $3 E, F, G, H$ ), the branch angles at the end of the vessels of cirrhotic livers were larger than the angles in the normal livers without overlapping. However, the qualitative and quantitative analyses of microvasculature might have been misled by superimposed vessel structures, especially in the area with rich vessels. Also, the bile ducts can be visualized in the samples from the BDL group, which are marked by yellow arrows in Figure 3D.

\section{PCCT images and 3D visualization of microvasculature}

The 3D surface reconstruction of microvasculature from the control group, the $\mathrm{CCl}_{4}$ group, the sham group, and the BDL group are presented in Figure 4. In the control group or sham group, the vascular tree has a regular shape and dichotomous branches. The vessels in the cirrhosis group are disordered and tortuous because of the compression of collagen tissues. Furthermore, the branching geometry of 

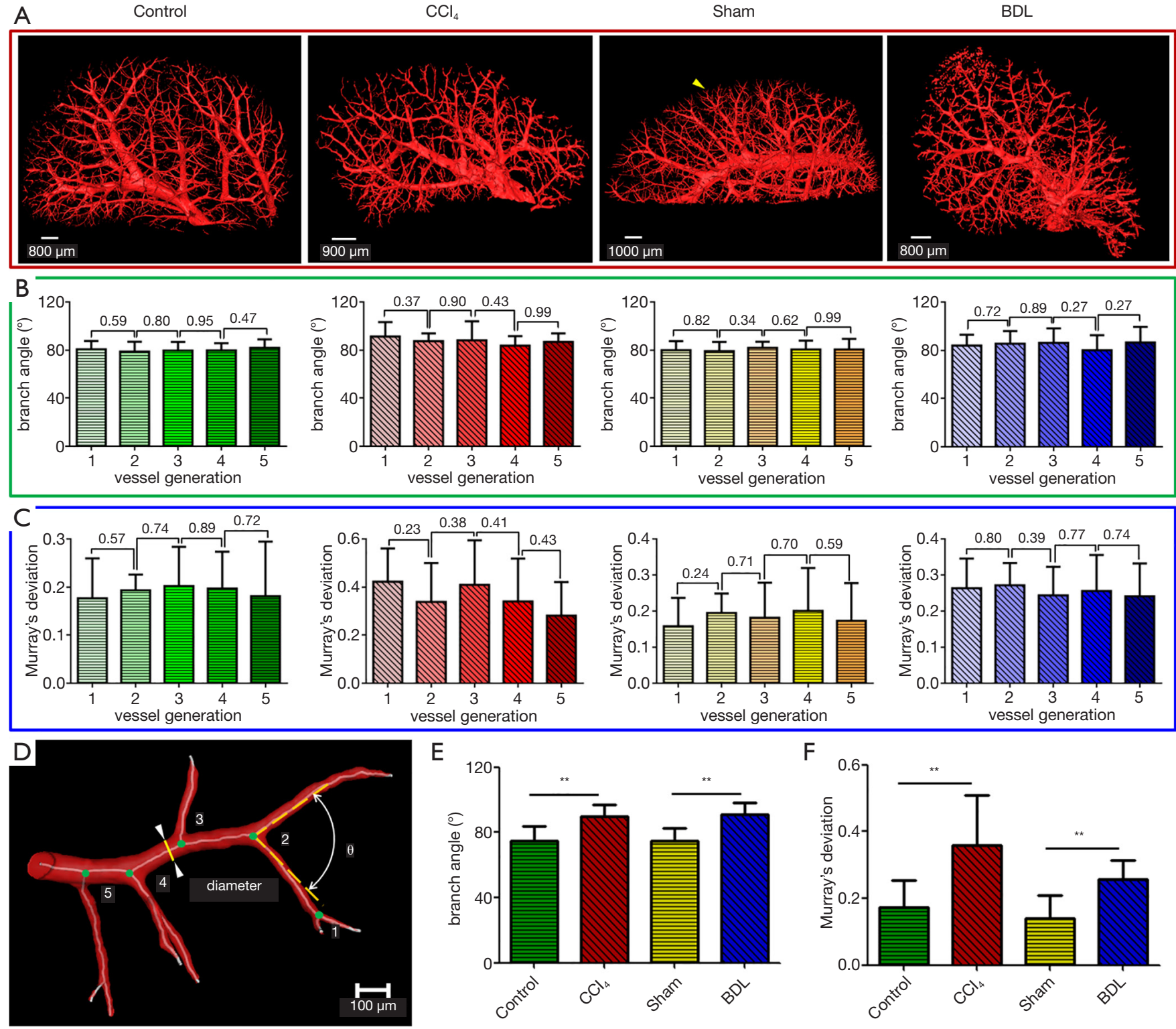

Figure 4 3D microvascular surface reconstructions and quantitative analyses. (A) The 3D microvasculatures of the control group, $\mathrm{CCl}_{4}$ group, sham group, and BDL group. The yellow arrow in (A) marked the finest blood vessel. The branch angle and MD of different generations in different groups are provided in (B) and (C), respectively (all P>0.20). (D) Schematic diagram of measurement of branch angle and vascular diameter. " $1-5$ " represents the different generations of branching from vascular ending to the main branch; " 1 " is the vascular ending, and the code of generations increases with the levels of branches. $\theta$ is the branch angle, and the white arrows indicate the measurement of diameter. The branch angle and $\mathrm{MD}$ are provided in (E) and (F) without consideration of vascular generations. Values are represented by means $\pm \mathrm{SD} .{ }^{* *} \mathrm{P}<0.01 . \mathrm{CCl}_{4}$, carbon tetrachloride; BDL, bile duct ligation; $\mathrm{MD}$, Murray's deviation.

the portal vein has changed. The quantitative analyses of the branch angle and MD in different groups were performed. Firstly, the branch angles and MDs from five generations of the chosen vascular segments were measured. There were not significant differences in MDs $(\mathrm{P}=0.23-0.89)$ or branch angles $(\mathrm{P}=0.27-0.99)$ in different generations (Figure $4 B, C)$. Finally, the branch angle and MD values in different groups were summarized when taking no account of vascular generations (Figure 4E,F). As shown in Figure $4 E$, the branch angles (mean $\pm \mathrm{SD}$ ) were significantly larger 
Table 1 Correlation between branch angle and MD with fibrosis area and portal pressure

\begin{tabular}{lccccc}
\hline \multirow{2}{*}{ Parameter } & \multicolumn{2}{c}{ Portal pressure } & & \multicolumn{2}{c}{ Fibrosis area } \\
\cline { 2 - 2 } \cline { 5 - 6 } & R value & $P$ & & R value & $P$ \\
\hline $\mathrm{CCl}_{4}$ model & & & & \\
Branch angle & 0.907 & $<0.01$ & 0.916 & $<0.01$ \\
MD & 0.849 & $<0.01$ & 0.807 & $<0.01$ \\
BDL model & & & & \\
Branch angle & 0.909 & $<0.01$ & 0.889 & $<0.01$ \\
MD & 0.821 & $<0.01$ & 0.761 & $<0.01$ \\
\hline $\begin{array}{l}\text { CCl } \\
\text { deviation. }\end{array}$ & & & &
\end{tabular}

in the $\mathrm{CCl}_{4}$ group and BDL group compared with those of the corresponding control groups $\left(89.99^{\circ} \pm 6.87^{\circ}\right.$ versus $74.83^{\circ} \pm 9.19^{\circ}, \mathrm{P}<0.01 ; 90.91^{\circ} \pm 7.39^{\circ}$ versus $74.82^{\circ} \pm 7.96^{\circ}$, $\mathrm{P}<0.01$, respectively). Also, the mean differences in branch angles were $15.16^{\circ} \pm 1.05^{\circ}\left(\mathrm{CCl}_{4}\right.$ versus control, $95 \% \mathrm{CI}$ : $13.09^{\circ}-17.22^{\circ}$ ) and $16.08^{\circ} \pm 0.99^{\circ}$ (BDL versus sham, $95 \%$ CI: $\left.14.13^{\circ}-18.04^{\circ}\right)$. In Figure $4 F$, the MDs in the $\mathrm{CCl}_{4}$ group and BDL group were $0.36 \pm 0.15$ and $0.26 \pm 0.06$ respectively, which were higher than the measurements obtained from the corresponding control groups $(0.17 \pm 0.08$ and $\mathrm{P}<0.01,0.14 \pm 0.07$ and $\mathrm{P}<0.01$, respectively). The mean differences in $\mathrm{MDs}$ were $0.19 \pm 0.02\left(\mathrm{CCl}_{4}\right.$ versus control, 95\% CI: $0.14-0.23$ ) and $0.12 \pm 0.01$ (BDL versus sham, $95 \%$ CI: 0.09-0.14).

\section{Relationship between portal pressure and branching geometric changes}

As shown in Table 1, a significant correlation was observed between $\mathrm{MD}$ and portal pressure in both the $\mathrm{CCl}_{4}$ model $(\mathrm{R}=0.849$ and $\mathrm{P}<0.01)$ and $\mathrm{BDL}$ model $(\mathrm{R}=0.821$ and $\mathrm{P}<0.01)$. Similar correlation between $\mathrm{MD}$ and fibrosis area was found in the $\mathrm{CCl}_{4}$ model $(\mathrm{R}=0.807, \mathrm{P}<0.01)$ and BDL model ( $\mathrm{R}=0.761, \mathrm{P}<0.01)$. Moreover, significant correlations were found between branch angle and portal pressure, as well as between branch angle and the fibrosis area in the $\mathrm{CCl}_{4}$ model $(\mathrm{R}=0.907$ and $\mathrm{P}<0.01, \mathrm{R}=0.916$ and $\mathrm{P}<0.01$, respectively) and BDL model $(\mathrm{R}=0.909$ and $\mathrm{P}<0.01$, $\mathrm{R}=0.889$ and $\mathrm{P}<0.01$, respectively).

The ROC curves were calculated from all rats. The area under the ROCs (AUROCs) of branch angle for the prediction of portal hypertension (portal pressure $\geq 10 \mathrm{mmHg}$ ) were 0.923 (95\% CI: $0.882-0.954$, cut-off value: $84.9^{\circ}$ ) with $81.7 \%$ sensitivity and $89.2 \%$ specificity in the $\mathrm{CCl}_{4}$ group (Figure $5 \mathrm{~A}$ ), and 0.949 (95\% CI: $0.913-0.973$, cut-off value: $84.9^{\circ}$ ) with $80.8 \%$ sensitivity and $95.8 \%$ specificity in the BDL group (Figure $5 B$ ). Additionally, the AUROCs for MD predictive of portal hypertension were 0.865 (95\% CI: $0.791-0.921$, cut-off value: 0.25$)$ with $83.3 \%$ sensitivity and $86.7 \%$ specificity in the $\mathrm{CCl}_{4}$ group (Figure $5 A$ ), and 0.896 (95\% CI: $0.827-$ 0.944 , cut-off value: 0.21 ) with $81.7 \%$ sensitivity and $85.0 \%$ specificity in the BDL group (Figure 5B).

\section{Discussion}

Microvascular changes in cirrhosis have been widely evaluated via histological sections. However, 3D microvascular features cannot be thoroughly analyzed by histology due to its 2D nature. In this study, PCCTbased $3 \mathrm{D}$ visualization techniques were used to realize the detection and characterization of morphological changes of microvasculature in $\mathrm{CCl}_{4}$-induced and $\mathrm{BDL}$-induced cirrhosis. The 3D morphology of the microvascular network was presented, and it provided more accurate branching patterns and geometric properties. Quantitative measurements of branching morphology features, such as branch angle and MD, were significantly higher in cirrhotic livers with portal pressure $\geq 10 \mathrm{mmHg}$ than in the normal group or the sham group (portal pressure $<10 \mathrm{mmHg}$ ). Furthermore, notable correlations were observed between the alterations of the fibrosis area and branching geometric parameters. According to ROC curves, branching geometric parameters were highly sensitive and specified diagnostic criteria for predicting portal hypertension.

Portal hypertension is mainly caused by increased intrahepatic vascular resistance and portal blood flow. Portal pressure measurement provides important prognostic information in patients with portal hypertension. To date, HVPG has been the most frequently adopted method to measure portal hypertension (28). However, HVPG measurement using catheterization on the hepatic vein requires a skilled interventional radiologist, which has limited the application in clinical practice (29). Thus, there are demands to explore new surrogate markers of HVPG that can discriminate portal pressure. Previous studies show that microvascular changes can be used to predict portal hypertension based on histological slices, and however, they are limited in 2D analyses, small fields of view and sampling errors $(4,30)$. PCCT has been used as a powerful tool to 

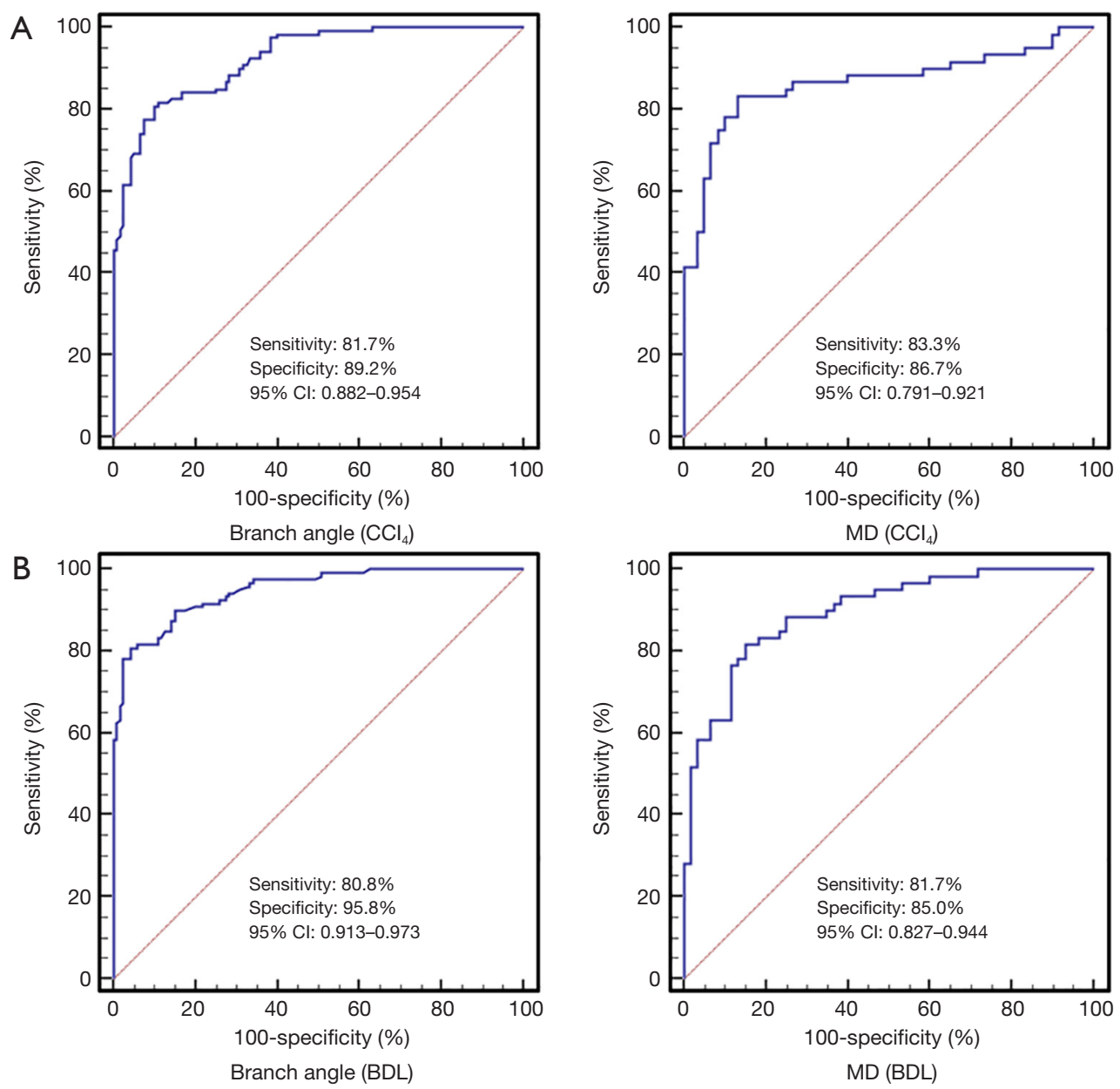

Figure 5 A depiction of the ROC curve analyses of branch angle and $\mathrm{MD}$ as predictors of significant portal hypertension in the $\mathrm{CCl}_{4}$ model (A) and BDL model (B). ROC, receiver operating characteristic; $\mathrm{CCl}_{4}$, carbon tetrachloride; $\mathrm{BDL}$, bile duct ligation; $\mathrm{MD}$, Murray's deviation.

reveal the $3 \mathrm{D}$ vascular structure and evaluate the changes in organ tissues $(3,16,17,31)$. In this study, the MD and branch angle in cirrhosis were measured, and significant correlations between branching geometric parameters and portal pressure were observed. The results demonstrate that the abnormal changes of microvascular branching morphology in cirrhosis were noteworthy factors for the prognosis of portal hypertension.

There still are some limitations in the present research. First, this study was performed ex vivo using cirrhotic samples of rats. More research is needed to evaluate whether the imaging patterns described herein apply for in vivo imaging as well. It is vital to reduce the radiation doses and scan time to achieve an in vivo application. The doses may be reduced by using optimized experimental instruments with increased X-ray energy, adequate spectrum filtering, and a highly efficient detector (32-35). Additionally, the long scan time can be further reduced using the new CT reconstruction algorithms (36,37). Recently, in vivo biomedical studies based on PCI and PCCT have become the subject of intense ongoing research $(38,39)$. Second, further evaluation will also be carried out in various types of liver fibrosis and increase experimental grouping in each model to validate the feasibility of branching geometric features in the differentiation of portal pressure. Third, PCCT could provide detailed $3 \mathrm{D}$ vascular morphologic information and acquire a larger field of view (FOV) than liver biopsy, but the FOV is still smaller than clinical CT. Recent research has shown that PCI could be available in commercial CT systems with a larger FOV (40). Also, the 
imaging of the smallest capillaries with sizes below $4 \mu \mathrm{m}$ is still limited by the resolution. However, a more improved spatial resolution may overcome this limitation, and then the corresponding segmentation and quantitative analysis can be performed.

In conclusion, this study confirms that quantitative analyses of branching morphologic characteristics, expressed as MD and branch angle, significantly correlated with portal pressure, suggesting the exceptional abilities of these parameters in the diagnosis of portal hypertension in cirrhosis. The branching morphologic features could be surrogate markers for detecting portal hypertension. Given the vast technical developments, it can be expected that in vivo PCCT will be available in the future, and thus this approach may serve as a potential non-invasive tool in diagnosing portal hypertension.

\section{Acknowledgments}

The authors would like to thank the staff from the beamline (BL13W1) of SSRF, China, for their kind assistance during the experiments.

Funding: This work was supported by the National Natural Science Foundation of China (Grant Nos. 81671683, 81670545 and 81371549), the Natural Science Foundation of Tianjin City in China (Grant No. 16JCYBJC28600), the Science and Technology Commission Foundation of Tianjin (Grant No. 2015KZ111), and the Open Project of Key Laboratory of Optoelectronic Information Technology, Ministry of Education (Tianjin University) (Grant No. 2017KFKT004).

\section{Footnote}

Conflicts of Interest: The authors have no conflicts of interest to declare.

Ethical Statement: The study protocol was approved by the Research Ethics Committee of Beijing Friendship Hospital, Capital Medical University, China.

\section{References}

1. Calvaruso V, Burroughs AK, Standish R, Manousou P, Grillo F, Leandro G, Maimone S, Pleguezuelo M, Xirouchakis I, Guerrini GP, Patch D, Yu D, O'Beirne J, Dhillon AP. Computer-assisted image analysis of liver collagen: relationship to Ishak scoring and hepatic venous pressure gradient. Hepatology 2009; 49:1236-44.

2. Annet L, Materne R, Danse E, Jamart J, Horsmans Y, Van Beers BE. Hepatic flow parameters measured with MR imaging and Doppler US: correlations with degree of cirrhosis and portal hypertension. Radiology 2003;229:409-14.

3. Xuan R, Zhao XY, Hu DD, Jian JB, Wang TL, Hu CH. Three-dimensional visualization of the microvasculature of bile duct ligation-induced liver fibrosis in rats by $\mathrm{X}$-ray phase-contrast imaging computed tomography. Sci Rep 2015;5:11500

4. Hu DD, Habib S, Li XM, Wang TL, Wang BE, Zhao XY. Angiogenesis: a new surrogate histopathological marker is capable of differentiating between mild and significant portal hypertension. Histol Histopathol 2015;30:205-12.

5. George SM, Eckert LM, Martin DR, Giddens DP. Hemodynamics in normal and diseased livers: application of image-based computational models. Cardiovasc Eng Technol 2015;6:80-91.

6. Kline TL, Zamir M, Ritman EL. Relating function to branching geometry: a micro-CT study of the hepatic artery, portal vein, and biliary tree. Cells Tissues Organs 2011;194:431-42.

7. Jonge ND, Ross FM. Electron microscopy of specimens in liquid. Nat Nanotechnol 2011;6:695-704.

8. San Lee J, Mook Weon B, Je JH. X-ray phase-contrast imaging of dynamics of complex fluids. J Phy D: Appl Phys 2013;46:494006.

9. Bruguier C, Mosimann PJ, Vaucher P, Uske A, Doenz F, Jackowski C, Mangin P, Grabherr S. Multiphase postmortem CT angiography: recognizing technique-related artefacts and pitfalls. Int J Legal Med 2013;127:639-52.

10. Bonse U, Busch F. X-ray computed microtomography (microCT) using synchrotron radiation (SR). Prog Biophys Mol Biol 1996;65:133-69.

11. Suortti P, Thomlinson W. Medical applications of synchrotron radiation. Phys Med Biol 2003;48:R1-35.

12. Hetterich H, Webber N, Willner M, Herzen J, Birnbacher L, Hipp A, Marschner M, Auweter SD, Habbel C, Schuller U, Bamberg F, Ertl-Wagner B, Pfeiffer F, Saam T. AHA classification of coronary and carotid atherosclerotic plaques by grating-based phase-contrast computed tomography. Eur Radiol 2016;26:3223-33.

13. Cao Y, Zhou Y, Ni SF, Wu TD, Li P, Liao SH, Hu JZ, Lu HB. Three dimensional quantification of microarchitecture and vessel regeneration by synchrotron radiation microcomputed tomography in a rat model of 
spinal cord injury. J Neurotrauma 2017;34:1187-99.

14. Mai C, Verleden SE, McDonough JE, Willems S, De Wever W, Coolen J, Dubbeldam A, Van Raemdonck DE, Verbeken EK, Verleden GM, Hogg JC, Vanaudenaerde BM, Wuyts WA, Verschakelen JA. Thin-section CT features of idiopathic pulmonary fibrosis correlated with micro-CT and histologic analysis. Radiology 2017;283:252-63.

15. Notohamiprodjo S, Webber N, Birnbacher L, Willner M, Viermetz M, Herzen J, Marschner M, Mayr D, Bartsch H, Saam T, Auweter S, Pfeiffer F, Reiser MF, Hetterich H. Qualitative and quantitative evaluation of structural myocardial alterations by grating-based phase-contrast computed tomography. Invest Radiol 2018;53:26-34.

16. Fu Y, Peng H, Zhang X, Peng WJ, Wu J, Wang SP, Du M, Li RM. Assessment of fibrotic tissue and microvascular architecture by in-line phase-contrast imaging in a mouse model of liver fibrosis. Eur Radiol 2016;26:2947-55.

17. Qin L, Zhao XY, Jian JB, Zhao YQ, Sun MY, Hu CH. High-resolution 3D visualization of ductular proliferation of bile duct ligation-induced liver fibrosis in rats using $\mathrm{X}$-ray phase contrast computed tomography. Sci Rep 2017;7:4215.

18. Zhang T, Xu XY, Zhou H, Zhao X, Song M, Zhang TT, Yin H, Li T, Li PT, Cai DY. A pharmacodynamic model of portal hypertension in isolated perfused rat liver. World J Gastroenterol 2012;18:472-8.

19. Zatroch KK, Knight CG, Reimer JN, Pang DSJ. Refinement of intraperitoneal injection of sodium pentobarbital for euthanasia in laboratory rats (Rattus norvegicus). BMC Vet Res 2017;13:60.

20. Bravin A, Coan P, Suortti P. X-ray phase-contrast imaging: from pre-clinical applications towards clinics. Phys Med Biol 2013;58:R1-35.

21. Chen RC, Rigon L, Longo R. Comparison of single distance phase retrieval algorithms by considering different object composition and the effect of statistical and structural noise. Opt Express 2013;21:7384-99.

22. Murray CD. The physiological principle of minimum work applied to the angle of branching of arteries. J Gen Physiol 1926;9:835-41.

23. Yang J, Yu LM, Sled JG, Henkelman RM. Comparative structural and hemodynamic analysis of vascular trees. Am J Physiol Heart Circ Physiol 2010;298:H1249.

24. Schoenenberger AW, Urbanek N, Toggweiler S, Seelos R, Jamshidi P, Resink TJ, Erne P. Deviation from Murray's law is associated with a higher degree of calcification in coronary bifurcations. Atherosclerosis 2012;221:124-30.
25. Xu P, Sasmito AP, Yu B, Mujumdar AS. Transport phenomena and properties in treelike networks. Appl Mech Rev 2016;68:040802.

26. Takahashi T. Microcirculation in fractal branching networks. Tokyo, Japan: Springer, 2014.

27. Youden WJ. Index for rating diagnostic tests. Cancer 1950;3:32-5.

28. Ripoll C, Groszmann R, Garcia-Tsao G, Grace N, Burroughs A, Planas R, Escorsell A, Garcia-Pagan JC, Makuch R, Patch D, Matloff DS, Bosch J. Hepatic venous pressure gradient predicts clinical decompensation in patients with compensated cirrhosis. Gastroenterology 2007;133:481-8.

29. Bureau C, Metivier S, Peron JM, Selves J, Robic MA, Gourraud PA, Rouquet O, Dupuis E, Alric L, Vinel JP. Transient elastography accurately predicts presence of significant portal hypertension in patients with chronic liver disease. Aliment Pharmacol Ther 2008;27:1261-8.

30. Castera L. Assessing liver fibrosis. Expert Rev Gastroenterol Hepatol 2008;2:541-52.

31. Duan J, Hu C, Chen H. High-resolution micro-CT for morphologic and quantitative assessment of the sinusoid in human cavernous hemangioma of the liver. PLoS One 2013;8:e53507.

32. Leong AFT, Buckley GA, Paganin DM, Hooper SB, Wallace MJ, Kitchen MJ. Real-time measurement of alveolar size and population using phase contrast X-ray imaging. Biomed Opt Express 2014;5:4024-38.

33. Meinel FG, Yaroshenko A, Hellbach K, Bech M, Müller M, Velroyen A, Bamberg F, Eickelberg O, Nikolaou K, Reiser MF, Pfeiffer F, Yildirim AÖ. Improved diagnosis of pulmonary emphysema using in vivo dark-field radiography. Invest Radiol 2014;49:653-8.

34. Guan Y, Wang Y, Yuan F, Lu H, Ren Y, Xiao T, Chen K, Greenberg DA, Jin K, Yang GY. Effect of suture properties on stability of middle cerebral artery occlusion evaluated by synchrotron radiation angiography. Stroke 2012;43:888-91.

35. Horng A, Brun E, Mittone A, Gasilov S, Weber L, Geith T, Adam-Neumair S, Auweter SD, Bravin A, Reiser MF, Coan P. Cartilage and soft tissue imaging using X-rays propagation-based phase-contrast computed tomography of the human knee in comparison with clinical imaging techniques and histology. Invest Radiol 2014;49:627-34.

36. Zanette I, Bech M, Rack A, Le Duc G, Tafforeau P, David C, Mohr J, Pfeiffer F, Weitkamp T. Trimodal low-dose X-ray tomography. Proc Natl Acad Sci U S A 2012;109:10199-204. 
37. Sidky EY, Anastasio MA, Pan XC. Image reconstruction exploiting object sparsity in boundary-enhanced X-ray phase-contrast tomography. Opt Express 2010;18:10404-22.

38. Moosmann J, Ershov A, Altapova V, Baumbach T, Prasad MS, LaBonne C, Xiao X, Kashef J, Hofmann R. X-ray phase-contrast in vivo microtomography probes new aspects of Xenopus gastrulation. Nature 2013;497:374-7.
39. Tang R, Li WX, Huang W, Yan F, Chai WM, Yang GY, Chen KM. CO2-based in-line phase contrast imaging of small intestine in mice. Sci Rep 2013;3:2313.

40. Khimchenko A, Schulz G, Thalmann P, Müller B. Implementation of a double-grating interferometer for phase-contrast computed tomography in a conventional system nanotom® m. APL Bioeng 2018;2:016106.

Cite this article as: Sun M, Lv W, Zhao X, Qin L, Zhao Y, Xin X, Jian J, Chen X, Hu C. Vascular branching geometry relating to portal hypertension: a study of liver microvasculature in cirrhotic rats by $\mathrm{X}$-ray phase-contrast computed tomography. Quant Imaging Med Surg 2020;10(1):116-127. doi: 10.21037/ qims.2019.11.04 
Supplementary

Table S1 Components and parameters of the PCCT setup

\begin{tabular}{ll}
\hline Component or parameter & Specification or value \\
\hline X-ray source & Synchrotron radiation \\
Photon energy (keV) & 24 \\
Source size & $408 \mu \mathrm{m} 23 \mu \mathrm{m}$ \\
Maximal beam size & $45 \mathrm{~mm}(\mathrm{H}) 5 \mathrm{~mm}(\mathrm{~V})$ \\
Monochromator & Si (111) double-crystal \\
Detector & 12 -bit charge-coupled- \\
& device camera* \\
Pixels & $4,0082,672$ \\
Pixel size ( $\left.\mu m^{2}\right)$ & 99 \\
Source-to-sample distance $(\mathrm{cm})$ & 34 \\
Sample-to-detector distance $(\mathrm{cm})$ & 80 \\
Projection images & $505-1,160$ \\
Exposure time per projection $(\mathrm{ms})$ & 12 \\
Exposure time per specimen $(\mathrm{min})$ & 9 \\
Dark-field images & 10 \\
Flat-field images & 10 \\
Image reconstruction & Filtered back-projection \\
\hline & algorithm \\
\hline
\end{tabular}

*, VHR1:1; Photonic Science, Ltd., Robertsbridge, UK. Additional details of the imaging system are described as follows: BL13W1 X-ray Imaging and Biomedical Applications Beamline. Web site: http://e-ssrf.sinap.cas.cn/beamlines/ bl13w1/201401/t20140112_152430.html. PCCT, phase-contrast computed tomography. 